\title{
New frontiers in aerodynamic tailoring of long span bridges: an advanced analysis framework
}

\author{
Xinzhong Chen*, Ahsan Kareem \\ NatHaz Modeling Laboratory, Department of Civil Engineering and Geological Sciences, \\ University of Notre Dame, 156 Fitzpatrick Hall, Notre Dame, IN 46556-0767, USA
}

\begin{abstract}
Significant developments in bridge aeroelastic analysis have been made utilizing realistic aerodynamic force modeling for bridges with bluff sections under turbulent winds. With these developments as a background, this paper highlights state-of-the-art developments in the aeroelastic analysis and identifies new frontiers in aerodynamic tailoring of long span bridges. Challenges in the aeroelastic analysis are pointed out that include: the modeling of aerodynamic forces excited by non-stationary wind fields such as hurricanes and thunderstorms and/or for bridges located in complex topography conditions; consideration of nonlinearities in both structural dynamics and aerodynamics; and the ubiquitous issues related to turbulence. In response to these challenges, an advanced analysis framework is offered that focuses on the needs for modeling of aerodynamic and structural nonlinearities, and the effects of turbulence on long span bridges.
\end{abstract}

(C) 2003 Elsevier Ltd. All rights reserved.

Keywords: Bridge aerodynamics; Wind load; Turbulence; Flutter; Buffeting; Bridges

\section{Introduction}

Long span bridges are very susceptible to the actions of strong wind. Earlier studies concerning long span bridge flutter was made by Bleich [1] utilizing airfoil flutter theory after the Tacoma Narrows Bridge disaster of 1940. By comparing bridge flutter with airfoil flutter, Selberg [2] suggested an empirical formulation for estimating the bridge flutter onset velocity. The pioneering work of Davenport [3] and Scanlan $[4,5]$, among others, concerning bridge buffeting and flutter have led to

*Corresponding author. Tel.: 219-631-4306; fax: 219-631-9236.

E-mail address: xchen@nd.edu (X. Chen). 
a number of analytical developments in bridge aerodynamics/aeroelasticity using realistic aerodynamic force modeling of bridges with bluff sections under turbulent winds (e.g., [6-14]). With these developments as a background, this paper highlights the state-of-the-art developments in the aeroelastic analysis and identifies new frontiers in aerodynamic tailoring of long span bridges. Challenges in the aeroelastic analysis are pointed out that include: the modeling of aerodynamic forces excited by non-stationary wind fields such as hurricanes and thunderstorms and/or for bridges located in complex topography conditions; consideration of nonlinearities in both structural dynamics and aerodynamics; and the ubiquitous issues related to turbulence. In response to these challenges, an advanced analysis framework is offered that focuses on the needs for modeling of aerodynamic and structural nonlinearities, and effects of turbulence on long span bridges.

\section{Modeling of linear unsteady aerodynamic forces}

The aerodynamic forces acting on bluff bridge sections can be represented for most cases by linear approximation and expressed in terms of time-averaged static, and time-varying self-excited and buffeting force components $[3,5]$. The aerostatic force components are characterized by static force coefficients; the unsteady selfexcited and buffeting force components are defined by flutter derivatives and admittance functions in the frequency domain, and by aerodynamic impulse response functions in the time domain. In addition, calculation of the integrated forces acting on a finite bridge element requires consideration of the spanwise coherence of forces in terms of joint acceptance functions, resulting in a reduction in the forces compared to those assumed to be spanwise fully coherent.

In the wind velocity range of interest for bridge design, the flow around bluff bridge sections is quite unsteady and not amenable to the quasi-steady theory, which neglects the unsteady fluid memory effect and is only valid at very high wind velocities. Therefore, consideration of unsteady aerodynamic characteristics are essential in the accurate modeling of forces and attendant aeroelastic response. Advances in the identification techniques for quantifying these aerodynamic forcing parameters, utilizing scaled bridge models in wind tunnels, have remarkably improved the accuracy in modeling aerodynamic forces and the prediction of aeroelastic response of overall bridges [15-21].

It has been widely understood that the uncoupled self-excited lift and drag, and pitching moment components, induced by the structural motion in respective directions, along with the coupled lift and pitching moment between vertical and torsional directions, are the most important terms in the prediction of aerodynamic damping and flutter behavior of a bridge. On the other hand, contributions of the coupled force components between lateral and torsional and between lateral and vertical directions have been considered to be less significant. However, experience of Akashi Kaikyo Bridge revealed that the coupled drag component due to torsional displacement played a significant role in the realization of negative aerodynamic damping at higher wind velocity range [22]. This was due to the unique aerodynamic 
feature of this truss section. The self-excited lift and pitching moment for this truss section were very small and the attendant aerodynamic damping was very low as wind velocity increased. In addition, large aerostatic drag force resulted in large negative angles of attack of the bridge section at which the aerostatic drag force exhibited large variations with respect to the angle of attack. These characteristics substantially increased the importance of the coupled drag component induced by the torsional displacement to the realization of negative aerodynamic damping. It is worth mentioning that, while it needs to be further confirmed, the contributions of coupled drag force components will remain insignificant in the case of bridge sections having low aerostatic drag and relatively large self-excited lift and pitching moment such as streamlined box sections.

Approximate inter-relationships among flutter derivatives and the relationship between flutter derivatives and admittance functions have been suggested [19,23,24], which helped to better understand the underlying mechanism and modeling of aerodynamic forces. These relationships were derived based on the approximate definition of the effective angle of incidence attributed to different structural motion components and wind fluctuations. Furthermore, their respective contribution to the aerodynamic forces could be uniquely quantified in terms of corresponding effective angle of incidence. It is noted that these relationships do not necessarily permit a plenary application to every bluff section. Therefore, their application to aeroelastic analysis should be handled carefully unless these are effectively validated. Continued discussion and experimental validation in this context are encouraged.

The frequency dependent aerodynamic force parameters are generally modeled in the frequency domain framework. However, most of the previous studies concerning time domain aeroelastic analysis were based on the quasi-steady assumption due to their inability to model frequency dependent unsteady aerodynamic characteristics in the time domain (e.g., [25]). In fact, these unsteady force characteristics can be described in terms of impulse response functions in the time domain. The time histories of unsteady aerodynamic forces can be calculated using convolution integrals involving the impulse response functions and structural motions or wind fluctuations (e.g., $[14,17])$.

Utilizing the relationship between both the time and frequency domain forcing functions and expressing the frequency domain forcing functions in terms of rational function approximations (RFAs) of the reduced frequency $k=\omega b / U$ (where $\omega$ is the circular frequency of motion; $U$ is the mean wind velocity; $B=2 b$ is the bridge deck width), time histories of unsteady aerodynamic forces corresponding to arbitrary structural motion and wind fluctuations can be calculated [14]. For example, the selfexcited lift per unit span, i.e., $L_{\text {seh }}(t)$, induced by a sinusoidal vertical motion, i.e., $h(t)=\bar{h}_{0} \mathrm{e}^{\mathrm{i} \omega t}$, is described by Scanlan and Tomko [4] and Scanlan [17]

$$
L_{\text {seh }}(t)=\frac{1}{2} \rho U^{2}(2 b)\left(k H_{1}^{*} \frac{\dot{h}}{U}+k^{2} H_{4}^{*} \frac{h}{b}\right),
$$

where $\rho$ is the air density; $H_{1}^{*}$ and $H_{4}^{*}$ are the flutter derivatives, which are functions of the reduced frequency and depend on the geometrical configuration of the bridge section and the approaching flow, and are defined at the statically deformed position 
of the bridge section under the action of aerostatic forces; and the over-dot denotes the partial differentiation with respect to time $t$.

In the time domain, $L_{\text {seh }}(t)$ corresponding to an arbitrary motion $h(t)$ is given by (e.g., $[14,17,26])$

$$
L_{\mathrm{seh}}(t)=\frac{1}{2} \rho U^{2} \int_{-\infty}^{t} I_{L h}(t-\tau) h(\tau) \mathrm{d} \tau,
$$

where $I_{L h}(t)$ is the aerodynamic impulse response function representing the influence of motion at a certain time instant on the generation of self-excited forces for a certain time period $t$.

Obviously, the relationship between $I_{L h}(t)$ and $H_{1}^{*}$ and $H_{4}^{*}$ is

$$
\bar{I}_{L h}=2 k^{2}\left(H_{4}^{*}+\mathrm{i} H_{1}^{*}\right),
$$

where $\bar{I}_{L h}$ is the Fourier transform of $I_{L h}(t)$.

The aerodynamic transfer function between $L_{\mathrm{seh}}(t)$ and $h(t)$ can be represented as following RFA of $k$ by curve-fitting the flutter derivatives at different reduced frequencies:

$$
2 k^{2}\left(H_{4}^{*}+\mathrm{i} H_{1}^{*}\right)=A_{L h, 1}+(\mathrm{i} k) A_{L h, 2}+(\mathrm{i} k)^{2} A_{L h, 3}+\sum_{j=1}^{m_{L h}} \frac{(\mathrm{i} k) A_{L h, j+3}}{\mathrm{i} k+d_{L h, j}},
$$

where $A_{L h, 1}, A_{L h, 2}, A_{L h, 3}, A_{L h, j+3}$ and $d_{L h, j}\left(d_{L h, j} \geqslant 0 ; j=1,2, \ldots, m_{L h}\right)$ are the frequency independent coefficients. The value of $m_{L h}$ determines the level of accuracy of this approximation and the size of additional equations representing the aerodynamic states.

Accordingly, $L_{\mathrm{seh}}(t)$ is given as a function of structural motion and a set of augmented aerodynamic states $\phi_{L h, j}(t)\left(j=1,2, \ldots, m_{L h}\right)$ as

$$
L_{\mathrm{seh}}(t)=-\frac{1}{2} \rho U^{2}\left(A_{L h, 1} h(t)+A_{L h, 2} \frac{b}{U} \dot{h}(t)+A_{L h, 3} \frac{b^{2}}{U^{2}} \ddot{h}(t)+\sum_{j=1}^{m_{L h}} \phi_{L h, j}(t)\right),
$$

where the aerodynamic states are given by a set of first order differential equations driven by the structural motion:

$$
\dot{\phi}_{L h, j}(t)=-\frac{d_{L h, j} U}{b} \phi_{L h, j}(t)+A_{L h, j+3} \dot{h}(t) \quad\left(j=1,2, \ldots, m_{L h}\right) .
$$

From the system theory viewpoint, the self-excited forces are modeled as the output of a system with structural response as the input. Their transfer functions are defined in terms of flutter derivatives. Similarly, the buffeting forces are derived as the output of a system with wind fluctuations as the input. Their transfer functions are described in terms of the admittance functions. To calculate the time histories of integrated forces over a given length of an element, modeling of a system characterized by the joint acceptance function is also required [14,27]. This framework offers a rigorous representation of the frequency dependent unsteady forces in the time domain provided that their unsteady characteristics can be represented by RFAs exactly or with an acceptable level of error. 
It is noted that other functions can also be chosen to express the aerodynamic transfer function as the function of reduced frequency $k$, provided that the selected function satisfies the causality requirement of the system or filter, i.e., the corresponding time response function of the system is zero at negative times. The condition that the real and imaginary parts of the transfer function are Hilbert pairs ensures this causality requirement. The causal transfer function model permits time domain simulation and it is physically realizable. The preceding RFA with positive parameters $d_{L h, j}\left(j=1,2, \ldots, m_{L h}\right)$ satisfies this causality requirement.

\section{Multimode coupled aeroelastic analysis}

Aerostatic analysis under time-averaged aerostatic forces is usually performed using a nonlinear iterative procedure taking into account both structural nonlinearity and nonlinear aerostatic forces which are functions of the angle of incidence. However, aerodynamic response around the aerostatic equilibrium can be analyzed using aerodynamic forces linearized at the statically deformed position of the bridge [5]. Akin to other structural dynamic problems, expressing structural equations of motion in terms of reduced-order structural modal coordinates is computationally effective. Scanlan [5] proposed the basic theory for multimode flutter and buffeting analyses. In practice, a mode-by-mode approach neglecting aerodynamic coupling among structural modes has widely been utilized based on the fact that practical flutter problems of long span bridges are most likely dampingdriven flutter and are dominated by the action of a single mode. Although this modeby-mode approach has proven its utility in many applications, experience shows that bridges with longer spans generally require a multimode coupled analysis framework (e.g., $[8,9,11,13,14,28,29,30])$.

The governing equations of dynamic motion of a bridge subjected to winds is expressed in terms of the generalized modal coordinates $\mathbf{q}$ as follows:

$$
\mathbf{M} \ddot{\mathbf{q}}+\mathbf{C} \dot{\mathbf{q}}+\mathbf{K q}=\mathbf{Q}_{\mathrm{se}}+\mathbf{Q}_{\mathrm{b}},
$$

where $\mathbf{M}, \mathbf{C}$ and $\mathbf{K}$ are the generalized mass, damping and stiffness matrices, respectively; $\mathbf{Q}_{\mathrm{se}}$ and $\mathbf{Q}_{\mathrm{b}}$ are the generalized self-excited and buffeting force vectors, respectively, and are expressed as

$$
\begin{aligned}
& \mathbf{Q}_{\mathrm{se}}=\frac{1}{2} \rho U^{2}\left(\mathbf{A}_{\mathrm{s}} \mathbf{q}+\frac{b}{U} \mathbf{A}_{\mathrm{d}} \dot{\mathbf{q}}\right), \\
& \mathbf{Q}_{\mathrm{b}}=\frac{1}{2} \rho U^{2}\left(\mathbf{A}_{\mathrm{bu}} \frac{\mathbf{u}}{U}+\mathbf{A}_{\mathrm{bw}} \frac{\mathbf{w}}{U}\right),
\end{aligned}
$$

where $\mathbf{A}_{\mathrm{s}}$ and $\mathbf{A}_{\mathrm{d}}$ are the aerodynamic stiffness and damping matrices, respectively; $\mathbf{A}_{\mathrm{bu}}$ and $\mathbf{A}_{\mathrm{bw}}$ are the buffeting force matrices; and $\mathbf{u}$ and $\mathbf{w}$ are the fluctuating wind vectors for the horizontal $u$ - and vertical $w$-components, respectively.

It has been a common practice to assume that low level of damping of motion does not influence generation of self-excited forces, therefore, $\mathbf{Q}_{\mathrm{se}}(t)$ corresponding to a 
sinusoidal motion $\mathbf{q}=\overline{\mathbf{q}} \mathrm{e}^{s t}$, where $s=(-\xi+\mathrm{i}) \omega$, can be expressed as

$$
\mathbf{Q}_{\mathrm{se}}(t)=\frac{1}{2} \rho U^{2}\left(\mathbf{A}_{\mathrm{s}}(\mathrm{i} k)+p \mathbf{A}_{\mathrm{d}}(\mathrm{i} k)\right) \overline{\mathbf{q}} \mathbf{e}^{s t}=\frac{1}{2} \rho U^{2}\left(\mathbf{A}_{\mathrm{s}}(\mathrm{i} k) \mathbf{q}+\frac{b}{U} \mathbf{A}_{\mathrm{d}}(\mathrm{i} k) \dot{\mathbf{q}}\right),
$$

where $\xi$ is the damping ratio; $p=s b / U=g+\mathrm{i} k$ and $g=-\xi k$. This method of dealing with damping in the aerodynamic parameters is referred to as $p-k$ method.

The equations of bridge motion can be represented in a state-space format having frequency dependent system matrix using the $p-k$ method. Consequently, the changes in modal frequencies, damping ratios and mode shapes as well as flutter behavior, with increasing wind velocity, can be estimated by the solution of a complex eigenvalue problem, omitting the buffeting force term. However, the solution at any given wind velocity needs an iterative calculation for each eigenvalue until the assumed frequency used to evaluate the self-excited aerodynamic forces agrees with that derived from the respective eigenvalue. In the case of closely spaced multimode flutter cases, potential changes in the order of frequencies with increasing wind velocity, does not permit full automation of the iterative target mode identification process, which makes this approach computationally cumbersome [13].

Generally, $\mathbf{Q}_{\mathrm{se}}(t)$ corresponding to $\mathbf{q}=\overline{\mathbf{q}}^{\mathrm{st}}$ can be given by the following $p$ domain-based expression that accounts for the influence of damping to the aerodynamic parameters:

$$
\mathbf{Q}_{\mathrm{se}}(t)=\frac{1}{2} \rho U^{2}\left(\mathbf{A}_{\mathrm{s}}(p)+p \mathbf{A}_{\mathrm{d}}(p)\right) \overline{\mathbf{q}} \mathrm{e}^{s t} .
$$

As the aerodynamic parameters are usually only available in the $k$ domain, i.e., corresponding to zero damping, the general form of aerodynamic parameters in the $p$ domain, i.e., corresponding to non-zero damping, can be approximated by assuming its analytic continuity in the domain of $g \geqslant 0$ and $g<0$ for small $g$. It can be realized by first expressing aerodynamic parameters in the $k$ domain in terms of a RFA of $k$ and then replacing $\mathrm{i} k$ by $p=g+\mathrm{i} k$ for those in the $p$ domain:

$$
\mathbf{A}_{\mathrm{s}}(\mathrm{i} k)+(\mathrm{i} k) \mathbf{A}_{\mathrm{d}}(\mathrm{i} k)=\mathbf{A}_{1}+(\mathrm{i} k) \mathbf{A}_{2}+(\mathrm{i} k)^{2} \mathbf{A}_{3}+\sum_{l=1}^{m} \frac{(\mathrm{i} k) \mathbf{A}_{l+3}}{\mathrm{i} k+d_{l}},
$$

where matrix $\mathbf{A}_{1}, \quad \mathbf{A}_{2}, \quad \mathbf{A}_{3}, \quad \mathbf{A}_{l+3}$ and $d_{l}\left(d_{l} \geqslant 0 ; l=l, 2, \ldots, m\right)$ are frequency independent coefficients, determined by curve-fitting aerodynamic matrix $\mathbf{A}_{\mathbf{s}}(\mathrm{i} k)+$ $(\mathrm{i} k) \mathbf{A}_{\mathrm{d}}(\mathrm{i} k)$.

Application of this $p$ method and incorporating the RFA technique result in frequency independent linear state-space equations of bridge motion with augmented aerodynamic states (e.g., [13]). This format leads to the calculation of all complex eigenvalues, at a given mean wind velocity, at the same time without iterative procedure. It becomes very attractive for a multimode flutter analysis with closely spaced frequencies. This format also facilitates a time domain simulation of the multimode flutter and buffeting responses. In addition, it provides a convenient format for the design of structural control schemes using modern control theory (e.g., [31]). 
Selection of participating structural modes in a multimode flutter analysis is usually not a difficult issue in practice. In fact, in most cases involving only a few important modes can provide an adequately accurate estimate of flutter behavior. The multimode approach offers more useful insight into the aerodynamic damping and inter-mode aerodynamic coupling that help to better understand the aeroelastic response. The role of each mode and the inter-modes coupled effects to system damping can be clarified from the viewpoint of the system energy variation [13]. The improved understanding of the significance of each structural mode in the aeroelastic response also offers useful information on the interpretation of wind tunnel-based full aeroelastic bridge model studies in which only a few important modes can be accurately modeled. The multimode approach is often computationally effective in comparison with the approach by directly using a finite-element bridge model (e.g., [8]).

Multimode coupled buffeting analysis has predominantly been conducted in the frequency domain using random vibration theory through spectral analysis. The transfer function matrix between the modal response $\mathbf{q}$ and generalized modal buffeting force $\mathbf{Q}_{\mathrm{b}}$ is given as

$$
\mathbf{H}_{\mathbf{q}}(\omega)=\left(-\omega^{2} \mathbf{M}+\mathrm{i} \omega\left(\mathbf{C}-\frac{1}{2} \rho U b \mathbf{A}_{\mathrm{d}}(\mathrm{i} k)\right)+\mathbf{K}-\frac{1}{2} \rho U^{2} \mathbf{A}_{\mathrm{s}}(\mathrm{i} k)\right)^{-1} .
$$

Conventional coupled buffeting analysis based on the evaluation of $\mathbf{H}_{\mathbf{q}}(\omega)$ using Eq. (13) requires matrix inversion at each discrete frequency which places a high computational demand. Such a time consuming procedure can be avoided by using a frequency independent state-space equations of an integrated system of the bridge and aerodynamics, or using a complex modal decomposition approach [32].

While the frequency domain approach facilitates modeling of frequency dependent characteristics of unsteady aerodynamic forces, it is limited to linear structural dynamics and aerodynamics and the assumption of stationarity of wind fluctuations. Challenges in aeroelastic analysis remain in the modeling of aerodynamic forces excited by non-stationary wind fields such as hurricane and thunderstorm and/or for bridges located in complex topography conditions [33-36], as well as consideration of nonlinearities in both structural dynamics and aerodynamics and the ubiquitous issue of turbulence [12,37,38]. Clearly, these challenges can only be adequately addressed in a time or time-frequency domain analysis framework.

Application of RFA technique for expressing unsteady aerodynamics facilitates the time domain simulation of unsteady forces. Utilizing the step-by-step numerical integration procedure, the aeroelastic response under the excitation of given time histories of spatiotemporal wind fluctuations can be simulated [14,32]. In addition, the aeroelastic bridge system can be further represented in an integrated state-space system driven by a vector-valued white noise, integrating characteristics of wind, aerodynamics and structural dynamics [27]. This framework allows direct calculations of the response covariance matrix using the Lyapunov equation that offers higher computational efficiency in comparison with conventional spectral analysis approach. 


\section{Multimode flutter: curve veering of frequency loci}

Fig. 1 shows the frequency and damping loci, as functions of mean wind velocity, for a wind-excited long span suspension bridge having a center span of nearly $2000 \mathrm{~m}$ with a streamlined box deck section. The flutter derivatives were calculated based on Theodorsen function. Analyses involving different structural modes indicated that the analysis involving structural modes 2,9,10, which are the fundamental vertical symmetric bending, second lateral symmetric bending and fundamental torsional symmetric modes, respectively, resulted in a flutter onset velocity of $65.3 \mathrm{~m} / \mathrm{s}$, which was close to $66.5 \mathrm{~m} / \mathrm{s}$ predicted when involving the first 15 structural modes. The eigenvalue loci of these complex mode branches were also similar, having a coupled flutter initiated from the complex mode branch 9 as indicated by solid lines. Without delving in a detailed discussion, this behavior may give the impression that the physics of the multimode coupled flutter is different from the general understanding of the coupled flutter in which two fundamental vertical and torsional structural modes are most important to flutter. This general understanding of the coupled flutter has been the foundation of both the preliminary bi-modal flutter prediction and wind tunnel study using spring-supported section models.

From the frequency and damping loci, it is observed that the eigenvalues of complex mode branches 9 and 10 repel each other without intersection at wind
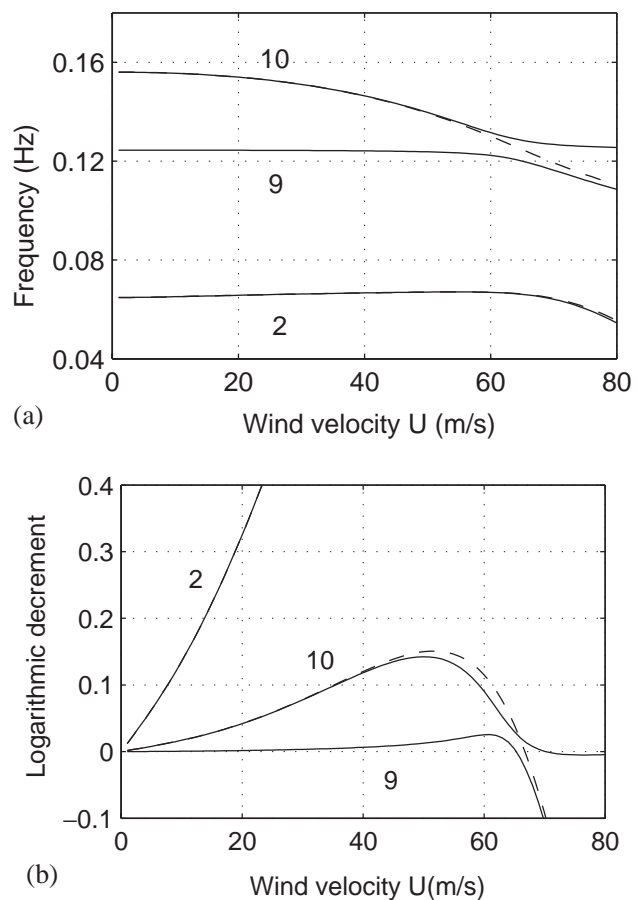

Fig. 1. Eigenvalue loci of the suspension bridge (-, w/structural modes $2,9,10 ;--, w /$ structural modes 2 and 10): (a) frequency vs. wind velocity and (b) damping ratio vs. wind velocity. 
velocities around $65 \mathrm{~m} / \mathrm{s}$. The complex mode shapes of these complex mode branches, in terms of the amplitude ratios of the structural mode components are given in Table 1. It is noted that the structural mode shapes were normalized in accordance with the maximum translateral displacement or torsional displacement multiplied by the half width of the bridge deck to be unity. It can be seen that both complex modes consist of strongly coupled components of the structural modes 9 and 10 with an additional coupled component of the structural mode 2 . As wind velocity exceeds $65 \mathrm{~m} / \mathrm{s}$, complex mode 9 gradually switches to a mode dominated by coupled components of structural modes 2 and 10. In the same way, complex mode 10 gradually changes to the mode dominated by the structural mode 9 . The eigenmodes (eigenvectors) associated with these two eigenvalues are exchanged continuously as if the curves of frequency and damping loci had intersected. This behavior has been termed the "curve veering phenomenon" [39]. Away from the veering region, the coupling of structural mode 9 with other modes becomes marginal.

Compared to the eigenvalues based on the two fundamental modes (structural modes 2 and 10) as shown in dashed lines in Fig. 1, it is evident that the underlying physics of the multimode coupled flutter involving more than two modes is essentially the same as the bi-modal coupled flutter, despite the fact that the flutter appears to initiate from different branches. The condition under which frequency loci veer was established and it was found that the curve veering was due to the influence of strong aerodynamic coupling among the adjacent structural modes [39].

Recent studies of coupled flutter using spring-supported bridge section models have shown that even in the case of bi-modal flutter, the heaving branch may also be the origin of so-called "heaving branch coupled flutter" [40]. From the viewpoint of curve veering, it was revealed that the heaving mode branch flutter corresponded to the intersection of frequency loci of heaving and torsional mode branches due to the weak influence of the coupled self-excited forces. It was noted that the heaving mode branch flutter was physically consistent with the generally observed torsional mode branch flutter [39].

Table 1

Amplitude ratios of the structural modes in the complex mode branches

\begin{tabular}{|c|c|c|c|c|c|c|}
\hline \multirow[t]{2}{*}{$U(\mathrm{~m} / \mathrm{s})$} & \multicolumn{3}{|c|}{ Mode branch 9} & \multicolumn{3}{|c|}{ Mode branch 10} \\
\hline & 2 & 9 & 10 & 2 & 9 & 10 \\
\hline 60 & 0.44 & $\underline{1.00}$ & 0.37 & 0.73 & 0.53 & $\underline{1.00}$ \\
\hline 62 & 0.60 & $\underline{1.00}$ & 0.49 & 0.79 & 0.71 & $\underline{1.00}$ \\
\hline 64 & 0.84 & $\underline{1.00}$ & 0.65 & 0.85 & 0.94 & $\underline{1.00}$ \\
\hline 66 & 1.15 & $\underline{1.00}$ & 0.84 & 0.90 & 1.22 & 1.00 \\
\hline 68 & 1.55 & 1.00 & $\underline{1.05}$ & 0.93 & $\underline{1.55}$ & 1.00 \\
\hline 70 & 2.04 & 1.00 & 1.27 & 0.96 & $\underline{1.91}$ & 1.00 \\
\hline 75 & 3.51 & 1.00 & $\underline{1.80}$ & 1.02 & $\underline{2.84}$ & 1.00 \\
\hline 80 & 5.18 & 1.00 & 2.22 & 1.05 & $\underline{3.68}$ & 1.00 \\
\hline
\end{tabular}




\section{Turbulence effects on flutter}

Wind tunnel-based flutter studies done with full-bridge aeroelastic models have often shown flutter onset velocity boundaries to be sharply defined in smooth flows whereas it exhibits rather gradual flutter boundaries in turbulent flows (e.g., [41]). A number of analytical studies based on randomizing the dynamic pressure and using stochastic techniques have been conducted to predict some general changes in the flutter instability due to turbulence in the approach flows (e.g., [26,42]). These studies tacitly assume that the mechanism that relates wind field to the aerodynamic forces remains unchanged in turbulent flow, which may not accurately represent the underlying physics [43,44]. Scanlan [45] explored the potential mechanism of turbulence on the single-mode torsional flutter due to a decrease in the spanwise correlation of the self-excited forces. Although the stabilizing effects of spanwise coherence loss may be apparent for single-mode flutter scenarios, it is not obvious that will apply to multimode coupled flutter cases. Coherence loss of self-excited forces along the span may stabilize a bridge flutter by reducing negative damping effects, and yet it may destabilize a bridge by reducing favorable damping effects. A recent experimental study supports full correlation of self-excited forces tacitly assumed in most current analytical approaches [46]. This implies that the turbulenceinduced changes in flutter instability of bridges cannot be explained entirely due to a decrease in the coherence of self-excited forces as noted in some studies.

\section{Nonlinear aerodynamic force model/aeroelastic analysis framework}

Current linear aerodynamic force models have proven their utility for a number of practical applications, however, these are not suited for addressing completely the challenges posed by aerodynamic nonlinearities and turbulence effects. Experimental studies have shown that the aerodynamic characteristics of many innovative bridge deck designs with attractive aerodynamic performance are very sensitive to the angle of incidence (e.g., [47,48]). For these bridge sections, even at low level of turbulence, the effective angle of incidence due to structural motion and incoming wind fluctuations may vary to a level such that the nonlinearities in the aerodynamic forces may not be neglected. Current linear analytical approaches also fall short in predicting the turbulence effects on the flutter boundary.

Diana et al. [12,37] proposed a nonlinear aerodynamic force model based on the so-called "quasi-static corrected theory," and they analytically investigated the turbulence effects on flutter and buffeting response. This nonlinear force model attempted to incorporate frequency dependent characteristics by decomposing the total response into components with different frequencies.

The present study concerns a detailed discussion of the nonlinear aerodynamic force model and the associated time domain analysis framework proposed by the authors for predicting the aeroelastic response of bridges under turbulent winds [38]. The nonlinear force model separates the forces into the low- and high-frequency components in accordance with the effective angle of incidence corresponding to the 
frequencies lower than and higher than a critical frequency, e.g., the lowest natural frequency of the bridge, i.e.

$$
\begin{aligned}
& \alpha_{\mathrm{e}}(t)=\alpha_{\mathrm{e}}^{1}(t)+\alpha_{\mathrm{e}}^{\mathrm{h}}(t), \\
& \mathbf{F}=\mathbf{F}\left(\alpha_{\mathrm{e}}\right) \approx \mathbf{F}\left(\alpha_{\mathrm{e}}^{1}\right)+\left.\frac{\mathrm{d} \mathbf{F}}{\mathrm{d} \alpha}\right|_{\alpha_{\mathrm{e}}^{1}} \alpha_{\mathrm{e}}^{\mathrm{h}}=\mathbf{F}^{\mathrm{l}}\left(\alpha_{\mathrm{e}}^{1}\right)+\mathbf{F}_{\mathrm{se}}^{\mathrm{h}}\left(\alpha_{\mathrm{e}}^{1}\right)+\mathbf{F}_{\mathrm{b}}^{\mathrm{h}}\left(\alpha_{\mathrm{e}}^{1}\right),
\end{aligned}
$$

where superscripts 1 and $\mathrm{h}$ indicate the low-frequency (including static component) and high-frequency components, respectively.

The low-frequency force component can be modeled based on the quasi-steady theory due to its high reduced velocity, while the high-frequency force component is separated into self-excited and buffeting force components which are modeled in terms of the frequency dependent unsteady aerodynamic characteristics at the lowfrequency spatiotemporally varying effective angle of incidence. For example, the high-frequency component of the self-excited lift force due to vertical motion is expressed as

$$
L_{\mathrm{seh}}^{\mathrm{h}}(t)=\frac{1}{2} \rho U^{2} \int_{-\infty}^{t} I_{L h}\left(\alpha_{\mathrm{e}}^{1}, t-\tau\right) h^{\mathrm{h}}(\tau) \mathrm{d} \tau,
$$

where the impulse response function is a function of both the effective angle of incidence and time. These are related to the flutter derivatives which are functions of both reduced frequency and angle of incidence.

The instantaneous low-frequency effective angle of incidence is determined based on the low-frequency component of turbulence and structural motion:

$$
\alpha_{\mathrm{e}}^{1}=\alpha_{\mathrm{s}}+\alpha^{1}+\phi^{1} ; \quad \phi^{1}=\tan ^{-1}\left(\frac{w^{1}+\dot{h}^{1}+0.5 b \dot{\alpha}^{1}}{U+u^{1}-\dot{p}^{1}}\right),
$$

where $\alpha_{\mathrm{S}}$ is the static angle of bridge deck under the action of aerostatic forces; and $p^{1}$ and $\alpha^{1}$ are low-frequency components of displacement in lateral and torsional directions, respectively.

When the low-frequency response is relatively small as is the case for long-span bridges, $\alpha_{\mathrm{e}}^{1}$ can be approximated by

$$
\alpha_{\mathrm{e}}^{1}=\alpha_{\mathrm{s}}+\tan ^{-1}\left(\frac{w^{1}}{U+u^{1}}\right) \approx \alpha_{\mathrm{s}}+\frac{w^{1}}{U+u^{1}} .
$$

The analysis framework using the proposed nonlinear aerodynamic force model is summarized in Fig. 2. Within this framework, the effects of low-frequency component of turbulence on flutter and buffeting responses can be analytically investigated. The effects of turbulence on flutter are modeled through the changes in the effective angle of incidence caused by turbulence and its influence on the selfexcited forces and the flutter instability.

Compared to the stochastic approach that relies simply on randomizing the dynamic pressure, which only includes the longitudinal wind fluctuations, this nonlinear framework incorporates a more physically meaningful influence of turbulence. The basic representation of the nonlinear force model is similar to that proposed by Diana et al. [12,37], however, this model provides a clear insight to the 


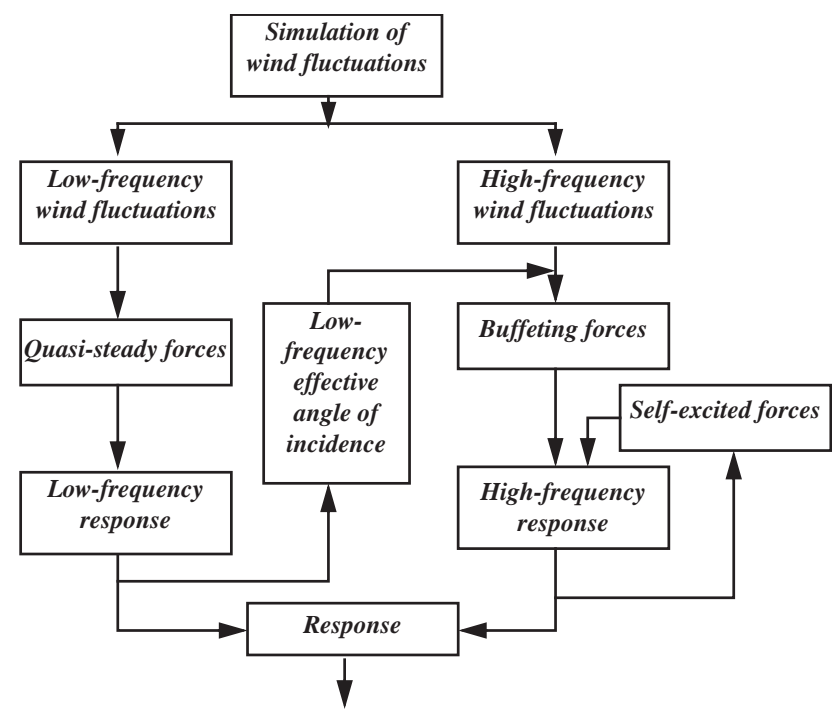

Fig. 2. The proposed nonlinear analysis framework.

relationship between the nonlinear and traditional linear force models, and presents a more efficient computational treatment by way of invoking rational function approximation to take into account the frequency dependent unsteady aerodynamic characteristics. The influence of high-frequency component of turbulence is manifested in terms of the changes in aerodynamic characteristics due to turbulence, which can be conveniently incorporated by employing aerodynamic characteristics derived in turbulent flows.

A long span suspension bridge with a main span of approximately $2000 \mathrm{~m}$ and a streamlined multibox deck section was used to illustrate the proposed nonlinear analysis framework and to investigate the influence of nonlinear aerodynamics and turbulence on the bridge response. The equations of bridge motion were described in terms of the first 15 structural modes with the natural frequencies ranging from 0.039 to $0.08 \mathrm{~Hz}$. The logarithmic decrement in each mode was assumed to be 0.02 .

Fig. 3 shows the flutter derivative $A_{2}^{*}$ at varying angles of incidence. For each angle of incidence, the flutter derivatives were expressed in terms of rational function approximation derived by a least-squares curve-fitting of the experimental data. The admittance and joint acceptance functions were considered not to vary with the angle of incidence for this example due to lack of experimental data. Although these features can be included in the proposed framework in a straightforward manner. Therefore, the nonlinearity in the buffeting forces was only introduced by the static force coefficients.

Fig. 4 shows variations of the damping ratio of a number of important complex mode branches, i.e., mode branches $1,2,8,9$, and 10, with the increasing wind velocity at the mean wind angles of incidence of $0^{\circ}$ and $\alpha_{\mathrm{s}}$. Modes 1 and 9 are the first and second symmetric lateral bending modes; modes 2 and 8 are the first and second 


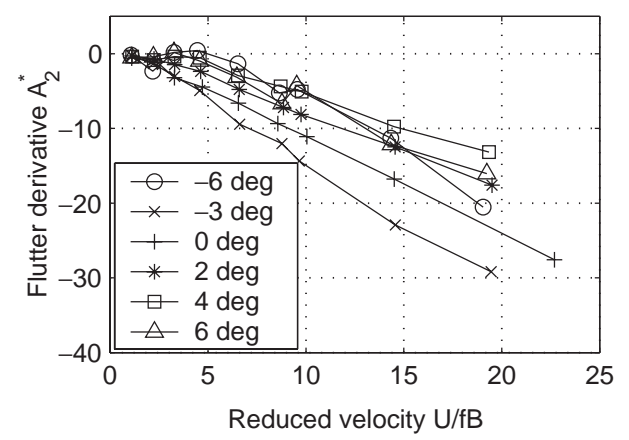

Fig. 3. Flutter derivatives $A_{2}^{*}$ at varying angles of incidence.

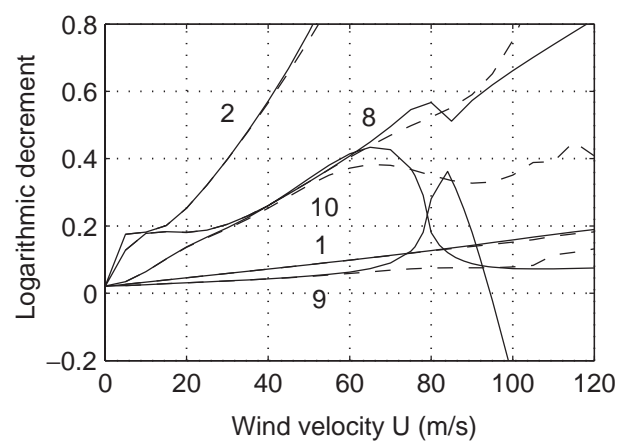

Fig. 4. Influence of static rotation of bridge deck on the aeroelastic damping $\left(-, 0^{\circ} ;--, \alpha_{\mathrm{s}}\right)$.

symmetric vertical bending modes; mode 10 is the first symmetric torsional mode. In the case of $0^{\circ}$, i.e., without the consideration of static deformation, the coupled flutter is predicted with a critical flutter velocity of $94.5 \mathrm{~m} / \mathrm{s}$ with a mode shape dominated by modes 2 and 10 . In the case in which the self-excited forces were linearized around the aerostatic equilibrium of the bridge corresponding to the angle of incidence of $\alpha_{\mathrm{s}}$, no flutter instability could be observed in the wind velocity range of up to $120 \mathrm{~m} / \mathrm{s}$. Remarkable static rotation of the bridge deck at high wind velocities resulted in a significant influence on the modal damping of the torsional mode dominated branch.

Results demonstrate the significance of including the changes in the aerodynamic force characteristics, with respect to the static angle of the bridge deck, for predicting accurately the aeroelastic response. This becomes even more important when the static rotation of bridge deck is remarkably large and the force characteristics are sensitive to the changes in the mean angle of incidence. On the other hand, it is noted that the results based on the nonlinear analysis are very close to the linear analysis based on the aerodynamic forces linearized at the statically deformed position of the bridge (Fig. 5). The insensitivity of the aerodynamic nonlinearities on the buffeting response may be due to the fact that the variations of the force characteristics with 


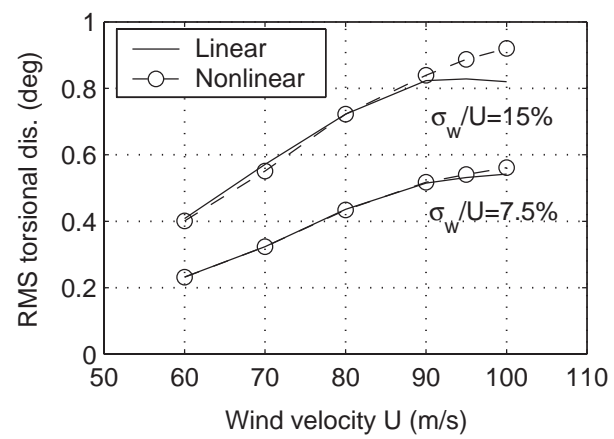

Fig. 5. Comparison of torsional buffeting response of the bridge deck.

the spatiotemporally varying effective angle of incidence did not result in an apparent build up or decay of the response, with inherent high aerodynamic damping. In addition, the spatial variation of the effective angle of incidence leads the modulation of the forces acting on different spanwise locations of the bridge deck to cancel one another such that the net effect due to changes in total forces on the bridge becomes less significant. This result does support the utility of the linear aerodynamic force model used in traditional analytical approaches.

It is noteworthy that the influence of aerodynamic nonlinearity depends on the level of the effective angle of incidence and the sensitivity of the force characteristics with respect to the effective angle of incidence. For the sake of comparison, nonlinear analysis without the inclusion of the static rotation of bridge deck in the effective angle of incidence was also conducted. The results were compared to those based on the linear analysis and shown in Fig. 6. The linear buffeting response demonstrates the existence of a flutter instability beyond $94.5 \mathrm{~m} / \mathrm{s}$ as predicted in the previous linear flutter analysis (Fig. 4). In the case of wind fluctuations having a turbulence intensity in the vertical direction of $\sigma_{\mathrm{w}} / U=7.5 \%$, a slight increase in flutter boundary is observed in the nonlinear analysis, while the responses are close to those based on the linear analysis. In the case of $\alpha_{\mathrm{w}} / U=15 \%$, the increase in the flutter boundary due to the nonlinear aerodynamic forces becomes very distinct. The aerodynamic nonlinearities with respect to the low-frequency effective angles of incidence apparently influence the buffeting response (Fig. 7), with inherent low aeroelastic damping, and stabilize the flutter instability. While the linear analysis always results in a distinct flutter boundary, the nonlinear analysis predicts a gradual increase in response with increasing wind velocity which is similar to the wind tunnel observations of full-aeroelastic bridge models under turbulent flows [41]. This suggests that the effects of turbulence on the flutter of full-bridges may be in part attributed to the aerodynamic nonlinearities, i.e. the nonlinearities in self-excited forces. The overall turbulence effects also include changes in aerodynamic characteristics due to turbulence which were not considered in the example study, but can be immediately included in the analysis when relevant data becomes available. 


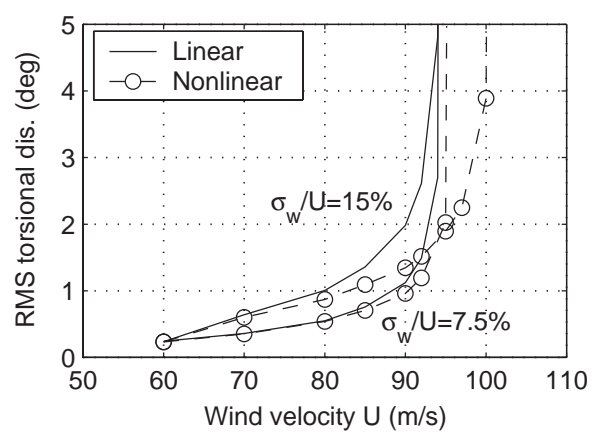

Fig. 6. Torsional buffeting response of the bridge deck neglecting the static deformation.

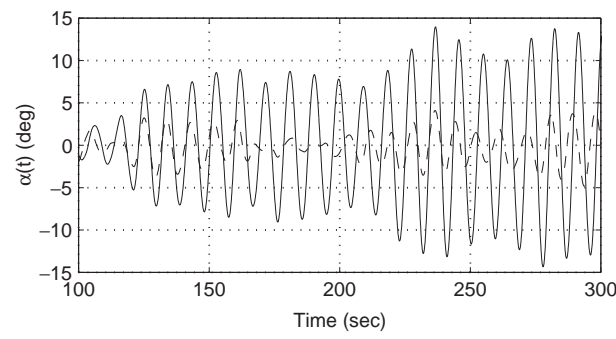

Fig. 7. Time histories of torsional displacement at the mid-point of the main span neglecting the static deformation $\left(U=95 \mathrm{~m} / \mathrm{s} ; \sigma_{\mathrm{w}} / U=15 \%\right.$, - , linear analysis; --, nonlinear analysis).

The authors would like to underscore that the proposed analysis framework is one of a few initial attempts to address the challenge of modeling nonlinearity and turbulence in aeroelastic response of bridges. While this study provides an effective analysis framework, additional follow-up studies, like those which followed the early development of linear bridge aeroelastic analysis, would help to refine or validate this approach.

\section{Concluding remarks}

This paper highlighted developments in the modeling of aerodynamic forces in both time and frequency domains including multimode coupled aeroelastic analysis of long span bridges. Needs and challenges in aeroelastic analysis were pointed out, including the modeling of aerodynamic forces excited by non-stationary wind fields such as hurricane and thunderstorm and/or for bridges located in complex topography conditions, consideration of nonlinearities in both structural dynamics and aerodynamics, and the ubiquitous issue of turbulence. These challenges can only be adequately addressed in a time or time-frequency domain analysis framework. An advanced nonlinear aerodynamic force model and attendant analysis framework 
were presented using an example long span bridge, focusing on the needs for modeling of aerodynamic and structural nonlinearities and effects of turbulence on long span bridges.

On a personal note, the second author would like to express his gratitude to Professor Davenport for his support, guidance and encouragement in his career. During recent international meetings the authors have noted with great interest the emphasis placed by Professor Davenport on the role of turbulence on the aerodynamics of bridges. In this context, the authors are very pleased to offer this analysis framework to help in unveiling the vexing complexities of turbulence effects on bridges.

\section{References}

[1] F. Bleich, Dynamic instability of truss-stiffened suspension bridges under wind action, Proc. ASCE 75 (3) (1949) 413-416 and 75(6) 855-865.

[2] A. Selberg, Oscillation and aerodynamic stability of suspension bridge. Acta Polytech. Scand. Civil Eng. Construct. Ser. 13 (1961).

[3] A.G. Davenport, Buffeting of a suspension bridge by stormy winds, J. Struct. Eng. ASCE 88 (ST3) (1962) 233-268.

[4] R.H. Scanlan, J.J. Tomko, Airfoil and bridge deck flutter derivatives, J. Eng. Mech. Div. ASCE 97 (EM6) (1971) 1717-1737.

[5] R.H. Scanlan, The action of flexible bridges under wind, 1: flutter theory; 2: buffeting theory, J. Sound Vib. 60 (2) (1978) 187-211.

[6] A.G. Davenport, J.P.C. King, Dynamic wind forces on long span bridges, Proceedings of the 12th IABSE Congress, Vancouver, Canada, 1984, pp. 705-712.

[7] J.P.C. King, A.G. Davenport, The determination of dynamic wind loads on long span bridges, Proceedings of the Fifth US National Conference on Wind Engineering, Lubbock, TX, November 1985, pp. 4A-25-4A-32.

[8] T. Miyata, H. Yamada, Coupled flutter estimate of a suspension bridge, International Colloquium on Bluff Body Aerodynamics and its Application, Kyoto, Japan, 1988, pp. 485-492.

[9] T.T.A. Agar, Aerodynamic flutter analysis of suspension bridges by a modal technique, Eng. Struct. 11 (1989) 75-82.

[10] R.H. Scanlan, N.P. Jones, Aeroelastic analysis of cable-stayed bridges, J. Struct. Eng. ASCE 116 (2) (1990) 279-297.

[11] N.P. Jones, R.H. Scanlan, A. Jain, H. Katsuchi, Advances (and challenges) in the prediction of longspan bridge response to wind, in: A. Larsen, S. Esdahl (Eds.), Bridge Aerodynamics, Balkema, Rotterdam, The Netherlands, 1998, pp. 59-85.

[12] G. Diana, S. Bruni, A. Collina, A. Zasso, Aerodynamic challenges in super long span bridges design, in: A. Larsen, S. Esdahl (Eds.), Bridge Aerodynamics, Balkema, Rotterdam, The Netherlands, 1998, pp. 131-144.

[13] X. Chen, M. Matsumoto, A. Kareem, Aerodynamic coupling effects on flutter and buffeting of bridges, J. Eng. Mech. ASCE 126 (1) (2000) 17-26.

[14] X. Chen, M. Matsumoto, A. Kareem, Time domain flutter and buffeting response analysis of bridges, J. Eng. Mech. ASCE 126 (1) (2000) 7-16.

[15] D.E. Walshe, T.A. Wyatt, Measurement and application of the aerodynamic admittance function for a box-girder bridge, J. Wind Eng. Ind. Aerodyn. 14 (1983) 211-222.

[16] A.G. Davenport, J.P.C. King, G. Larose, Taut strip model test, in: A. Larsen (Ed.), Aerodynamics of Large Bridges, Balkema, Rotterdam, The Netherlands, 1992, pp. 113-124.

[17] R.H. Scanlan, Problematics in formulation of wind-force models for bridge decks, J. Eng. Mech. ASCE 119 (7) (1993) 1353-1375. 
[18] P.P. Sarkar, N.P. Jones, R.H. Scanlan, Identification of aeroelastic parameters of flexible bridges, J. Eng. Mech. ASCE 120 (8) (1994) 1718-1742.

[19] M. Matsumoto, Y. Nihara, Y. Kobayashi, H. Shirato, H. Hamasaki, Flutter mechanism and its stabilization of bluff bodies, Proceedings of the Ninth International Conference on Wind Engineering, New Delhi, India, 1995, pp. 827-838.

[20] G.L. Larose, J. Mann, Gust loading on streamlined bridge decks, J. Fluids Struct. 12 (1998) 511-536.

[21] X. Chen, A. Kareem, Advances in the modeling of aerodynamic forces on bridge decks, J. Eng. Mech. ASCE 128(11) (2002).

[22] T. Miyata, K. Tada, H. Sato, H. Katsuchi, Y. Hikami, New findings of coupled flutter in full model wind tunnel tests on the Akashi Kaikyo Bridge, Proceedings of the Symposium on Cable-Stayed and Suspension Bridges, Deauville, France, October 12-15, 1994, pp. 163-170.

[23] R.H. Scanlan, N.P. Jones, L. Singh, Inter-relation among flutter derivatives, J. Wind Eng. Ind. Aerodyn. 69-71 (1997) 829-837.

[24] R.H. Scanlan, N.P. Jones, A form of aerodynamic admittance for use in bridge aeroelastic analysis, J. Fluids Struct. 13 (1999) 1017-1027.

[25] T. Miyata, H. Yamada, V. Boonyapinyo, J.C. Stantos, Analytical investigation on the response of a very long suspension bridge under gusty wind, Proceedings of the Ninth International Conference on Wind Engineering, New Delhi, India, 1995, pp. 1006-1017.

[26] C.G. Bucher, Y.K. Lin, Stochastic stability of bridges considering coupled modes, J. Eng. Mech. ASCE 114 (12) (1989) 2055-2071.

[27] X. Chen, A. Kareem, Aerodynamic analysis of bridges under multi-correlated winds: integrated statespace approach, J. Eng. Mech. ASCE 127 (11) (2001) 1124-1134.

[28] Y.J. Ge, H. Tanaka, Aerodynamic stability of long-span suspension bridges under erection, J. Struct. Eng. ASCE 126 (12) (2000) 1404-1412.

[29] H. Xiang, A. Chen, Aerodynamic studies of long-span cable-supported bridges in China, in: J.M. Ko, Y.L. Xu (Eds.), Advances in Structural Dynamics, Vol. 1, Elsevier Science Ltd., Amsterdam, 2000, Proceedings of International Conference on Advanced Structural Dynamics, Hong Kong, China, pp. 121-132.

[30] Y.L. Xu, D.K. Sun, J.M. Ko, J.H. Lin, Fully coupled buffeting analysis of Tsing Ma suspension bridge, J. Wind Eng. Ind. Aerodyn. 85 (1) (2000) 97-117.

[31] K. Wilde, Y. Fujino, J. Masukawa, Time domain modeling of bridge deck flutter, J. Struct. Mech. Earthq. Eng. (Japan Society of Civil Engineers) 13(2) (1996) 93-104.

[32] X. Chen, A. Kareem, Advanced analysis of coupled buffeting response of bridges: a complex modal decomposition approach, Probab. Eng. Mech. 17 (2002) 201-213.

[33] A.G. Davenport, J.P.C. King, The influence of topography and storm structure on long span bridge behavior in wind, International Seminar on Utilization of Large Boundary Layer Wind Tunnels. Public Works Institute, Japan, 1993, pp. 11-22.

[34] T. Miyata, H. Sato, R. Toriumi, Y. Otani, Experimental study on gust response of cable stayed bridge in complicated topography, Proceedings of 14th National Symposium on Wind Engineering, Tokyo, Japan, Japan Association for Wind Engineering, 1996, pp. 521-526 (in Japanese).

[35] A. Honda, S. Fukahori, S. Imagane, F. Ishioka, Aerodynamic design of cable-stayed bridge surrounded by undulated topography, in: A. Larsen, G.L. Larose, F.M. Livesey (Eds.), Wind Engineering into 21st Century, Balkema, Rotterdam, The Netherlands, 1999, pp. 913-926.

[36] C.W. Letchford, C. Mans, M.T. Chay, Thunderstorms - their importance in wind engineering, a case for the next generation wind tunnel, J. Wind Eng., Proceedings of the Fifth Asia-Pacific Conference on Wind Engineering (APCWE V), Vol. 89, Kyoto, Japan, Japan Association for Wind Engineering, 2001, pp. 31-43.

[37] G. Diana, F. Cheli, A. Zasso, M. Bocciolone, Suspension bridge response to turbulent wind: comparison of new numerical simulation method results with full scale data, in: A. Larsen, G.L. Larose, F.M. Livesey (Eds.), Wind Engineering into the 21 Century, Balkema, Rotterdam, The Netherlands, 1999, pp. 871-878.

[38] X. Chen, A. Kareem, Nonlinear response analysis of long-span bridge under turbulent winds, J. Wind Eng. Ind. Aerodyn. 89 (14-15) (2001) 1335-1350. 
[39] X. Chen, A. Kareem, Curve veering of eigenvalue loci of bridges with aeroelastic effects, J. Eng. Mech. ASCE (2002) 129 (2) (2003) 146-159.

[40] M. Matsumoto, F. Yoshizumi, T. Yabutani, K. Abe, N. Nakajima, Flutter stabilization and heavingbranch flutter, J. Wind Eng. Ind. Aerodyn. 83 (1999) 283-299.

[41] H.P.A.H. Irwin, Wind tunnel and analytical investigation of the response of Lions Gate Bridge to turbulent wind, NRC of Canada, NAE-LTR-LA-210, 1977.

[42] Y.K. Lin, Q.C. Li, New stochastic theory for bridge stability in turbulent flow, J. Eng. Mech. ASCE 119 (1) (1993) 113-127.

[43] Y. Nakamura, Bluff-body aerodynamics and turbulence, J. Wind Eng. Ind. Aerodyn. 49 (1993) $65-78$.

[44] M. Matsumoto, Recent study on bluff body aerodynamics and its mechanism, in: A. Larsen, G.L. Larose, F.M. Livesey (Eds.), Wind Engineering into the 21 Century, Balkema, Rotterdam, The Netherlands, 1999, pp. 67-78.

[45] R.H. Scanlan, Amplitude and turbulence effects on bridge flutter derivatives, J. Struct. Eng. ASCE 123 (2) (1997) 232-236.

[46] F.L. Haan, A. Kareem, A.A. Szewczyk, Experimental measurements of spanwise correlation of selfexcited forces on a rectangular cross section, Volume of Abstracts, Fourth International Colloquium on Bluff Body Aerodynamics and Applications (BBAA IV), Germany, 2000, pp. 439-442.

[47] A. Zasso, A. Curami, Extensive identification of bridge deck aeroelastic coefficient: average angle of attack, Reynolds number and other parameters effects, Proceedings of the Third Asian Pacific Symposium on Wind Engineering, Hong Kong, China, 1993, pp. 143-148.

[48] M. Matsumoto, T. Yagi, H. Ishizaki, H. Shitato, X. Chen, Aerodynamic stability of 2-edge girders for cable-stayed bridge, Proceedings of 15th National Symposium on Wind Engineering, Tokyo, Japan, Japan Association for Wind Engineering, 1998, pp. 389-394 (in Japanese). 